\title{
Thermal-Fluid Transport Phenomena of a Strongly-Heated Gas Flow in Parallel Tube Rotation
}

\author{
SHUICHI TORII ${ }^{\mathrm{a}, *}$ and WEN-JEI YANG ${ }^{\mathrm{b}}$ \\ ${ }^{a}$ Department of Mechanical Engineering, Kagoshima University, 1-21-40 Korimoto, Kagoshima 890, Japan; \\ ${ }^{\mathrm{b}}$ Department of Mechanical Engineering and Applied Mechanics, University of Michigan, Ann Arbor, MI 48109, USA
}

(Received 15 January 1998; Revised 5 February 1998)

\begin{abstract}
A numerical analysis is performed to study thermal transport phenomena in gas flow through a strongly heated tube whose axis is in parallel with the rotational axis. The velocity and temperature fields prevail when fluid flows in a rotating tube with uniform heat flux on the tube wall. The two-equation $k-\varepsilon$ turbulence and $\frac{g}{t^{2}}-\varepsilon_{t}$ heat transfer models are employed to determine turbulent viscosity and eddy diffusivity for heat, respectively. The governing boundary-layer equations are discritized by means of a control volume finitedifference techniques. It is found that the Coriolis and centrifugal (or centripetal) forces cause fluid flow and heat transfer performance in the parallel-rotation system to be drastically different from those in the stationary case. Consequently, even if a tube rotating around a parallel axis is heated with high heat flux whose level causes a laminarizing flow in the stationary tube case, both the turbulent kinetic energy and the temperature variance remain over the pipe cross section, resulting in the suppression of an attenuation in heat transfer performance. In other words, an increase in tube rotation suppresses laminarization of gas flow.
\end{abstract}

Keywords: Thermal transport phenomena, Parallel rotation, Turbulent kinetic energy, Temperature variance, Turbulent heat flux

\section{INTRODUCTION}

An effect of channel rotation on flows in rotating machinery, such as flows in a rotary pump, high temperature gas turbine, high power electric generators, motors and other rotating systems, has been investigated. However, its roles in flow and heat transfer mechanisms are not yet fully understood, because the flow and heat transfer mechanisms in the rotating turbine blades are extremely complex due to the presence of both the Coriolis force and the buoyancy force in the centrifugal acceleration field. Generally, the engineering applications can be classified into four types of rotation or revolution, i.e. radial rotation, parallel revolution, axial rotation and

*Corresponding author. Tel.: 099/285-8245. Fax: 099/250-3181. E-mail: torii@mech.Kagoshima-u.ac.jp. 
circumferential revolution (Yang and Zhang, 1992). The coaxial rotating duct is a special case of the parallel revolving duct without eccentricity. In accordance with the applications of heat transfer to the advanced rotating machineries, it is necessary and important to understand the heat transfer processes in rotating channels. The detailed information on the heat transfer characteristics in rotating channels is valuable in the design and calculation of cooling system in the rotating machineries. Experimental and theoretical studies on transport phenomena in rotating and revolving pipes or ducts were performed by numerous authors, for example, Mori and Nakayama (1984), Kataoka et al. (1984), Mori and Nakayama (1968), Mori et al. (1971). Morris (1981) reviewed the known information on the influence of rotation on flow and heat transfer in ducts which are constrained to rotate about a prescribed axis. The present study is focused on flows through a tube, whose axis is in parallel with the rotational axis.

When a gas in a channel is heated with extremely high heat flux, the flow may possibly be laminarized, that is, a transition from turbulent to laminar flows occurs at a higher Reynolds number than the usual critical value, i.e. $\operatorname{Re}=2300$. This phenomenon is referred to as laminarization. The experimental and numerical studies on the thermal fluid transport phenomena have been performed by several investigators: Bankston (1970), Coon and Perkins (1970), McEligot et al. (1970), Perkins et al. (1973), Mori and Watanabe (1979), Ogawa et al. (1982), Kawamura (1979). Torii et al. (1993) and Torii and Yang (1997a) investigated the transport phenomena in the strongly heated circular tube flows by means of a Reynolds stress turbulence model and a two-equation heat transfer model, respectively. It is disclosed that (i) even when a substantial reduction in heat transfer, i.e. laminarization takes place, the turbulent kinetic energy does not disappear completely, (ii) laminarization due to strong heating causes both a reduction in the Reynolds stress and an amplification of the inherent anisotropy of turbulence structure, (iii) both the temperature variance and the turbulent heat flux are also diminished over the whole tube cross section in the flow direction, and (iv) although both the velocity dissipation time scale and the temperature dissipation time scale are substantially amplified in the laminarizing flow, their ratio is slightly increased. Torii and Yang (1997b) reported the thermal transport phenomena in a strongly heated gas flow in a circular tube rotating around the axis. It is found that (i) the presence of tube rotation contributes to the promotion of laminarization of gas flow, and (ii) a reduction in the velocity gradient induced by tube rotation suppresses the production of turbulent kinetic energy, resulting in an amplification in laminarizing the flow process. In contrast, to the authors' knowledge, there is no detailed experimental data pertinent to flow and thermal characteristics such as turbulence quantities in a strongly heated pipe in parallel rotation, because of the complexity of the fluid flow and the difficulty of the measurement. Numerical analysis is a useful method of studying fluid flow and heat transfer in the tube, whose axis is in parallel with the rotational axis. As for the existing theoretical study, the assumption of constant properties is employed in the governing equations to be solved, although the coolant in the rotating machinery, particularly in the high temperature gas turbine, is imposed with high heat flux heating.

The purpose of the present study is to investigate a strongly heated gas flow in a circular tube, whose axis is in parallel with the rotational axis. The $\overline{t^{2}}-\varepsilon_{t}$ heat transfer model proposed by Torii and Yang (1997a) and the $k-\varepsilon$ turbulence model of Torii et al. (1990) are employed to determine the mechanism of heat transport phenomena. The turbulent thermal conductivity $\lambda_{t}$ is determined using the temperature variance, $\bar{t}^{2}$, and the dissipation rate of temperature fluctuations, $\varepsilon_{t}$, together with $k$ and $\varepsilon$. Emphasis is placed on effects of parallel rotation of the pipe on the flow and thermal fields, i.e. turbulent kinetic energy, streamwise velocity, and temperature variance. 


\section{GOVERNING EQUATIONS AND NUMERICAL SCHEME}

A turbulent flow in a strongly heated circular tube whose axis is in parallel with the rotational axis, as shown in Fig. 1, is analyzed using cylindrical coordinates. In this analysis, the dependence of gas properties on temperature as well as change in gas density must be taken into account (Schlichting, 1985). Here, the derivation processes of the governing equations are expressed in reference (Schlichting, 1985). The following assumptions are imposed in the formulation of the problem: an incompressible, steady flow, negligible viscous heating and neglect of viscous dissipation in the energy equation. Thus, the governing equations read

$$
\begin{aligned}
\frac{\partial \bar{\rho} U_{i}}{\partial x_{i}} & =0 \\
\bar{\rho} U_{j} \frac{\partial U_{i}}{\partial x_{j}} & =-\frac{\partial P}{\partial x_{i}}+\frac{\partial}{\partial x_{j}}\left(\mu \frac{\partial U_{i}}{\partial x_{j}}-\bar{\rho} \overline{u_{i} u_{j}}\right)+F \\
c_{p} \bar{\rho} U_{i} \frac{\partial T}{\partial x_{i}} & =\frac{\partial}{\partial x_{i}}\left(\lambda \frac{\partial T}{\partial x_{i}}-c_{p} \bar{\rho} \overline{u_{i} t}\right) .
\end{aligned}
$$

Here, a third term, $F$, in Eq. (2) implies Coriolis forces and centrifugal buoyancy forces caused in the flow and temperature fields, which are summarized in Table I for each component. The Reynolds stress $-\bar{\rho} \overline{u_{i} u_{j}}$ in Eq. (2) is obtained by using the

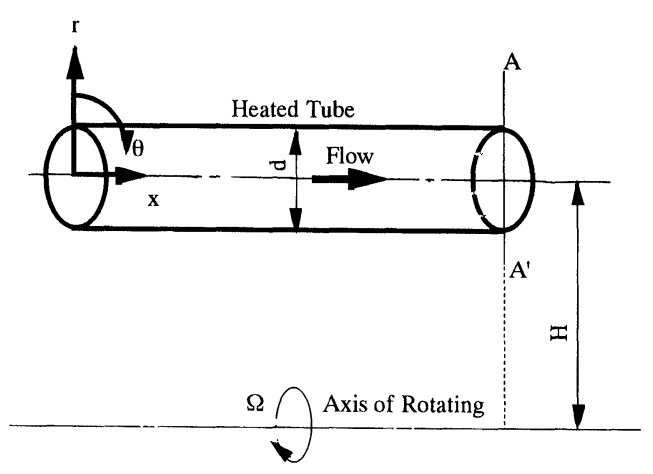

FIGURE 1 A schematic of physical system and coordinate.
TABLE I Coriolis forces and centrifugal buoyancy forces caused in the flow and temperature fields

\begin{tabular}{ll}
\hline Component & \multicolumn{1}{c}{$F$} \\
\hline$r$ & $2 \rho \Omega W+\rho \Omega^{2} \beta(r+H \cos \theta)\left(T_{\mathrm{w}}-T_{\mathrm{b}}\right)$ \\
$\theta$ & $-2 \rho \Omega V-\rho \Omega^{2} \beta\left(T_{\mathrm{w}}-T_{\mathrm{b}}\right) \sin \theta$ \\
$x$ & 0 \\
\hline
\end{tabular}

Boussinesq approximation

$$
-\bar{\rho} \overline{u_{i} u_{j}}=\mu_{t}\left(\frac{\partial U_{i}}{\partial x_{j}}+\frac{\partial U_{j}}{\partial x_{i}}\right)-\frac{2}{3} \delta_{i j} \bar{\rho} k .
$$

Here, the turbulent viscosity $\mu_{t}$ can be expressed in terms of the turbulent kinetic energy $k$ and its dissipation rate $\varepsilon$, referring to the KolmogorovPrandtl's relation (Rodi, 1982), as

$$
\mu_{t}=C_{\mu} f_{\mu} \bar{\rho} \frac{k^{2}}{\varepsilon} .
$$

$C_{\mu}$ and $f_{\mu}$ are a model constant and a model function, respectively. Torii et al. (1990) developed the low Reynolds number version of the $k-\varepsilon$ turbulence model capable of reproducing the transition from turbulent to laminar flows, which was originally developed by Nagano and Hishida (1987). The same model is employed here. Both transport equations read

$$
\begin{aligned}
\bar{\rho} U_{j} \frac{\partial k}{\partial x_{j}}= & \frac{\partial}{\partial x_{j}}\left\{\left(\mu+\frac{\mu_{t}}{\sigma_{k}}\right) \frac{\partial k}{\partial x_{j}}\right\} \\
& -\bar{\rho} \overline{u_{i} u_{j}} \frac{\partial U_{i}}{\partial x_{j}}-\bar{\rho} \varepsilon-2 \mu\left(\frac{\partial \sqrt{k}}{\partial x_{i}}\right)^{2}, \\
\bar{\rho} U_{j} \frac{\partial \varepsilon}{\partial x_{j}}= & \frac{\partial}{\partial x_{j}}\left\{\left(\mu+\frac{\mu_{t}}{\sigma_{\varepsilon}}\right) \frac{\partial \varepsilon}{\partial x_{j}}\right\} \\
& -C_{1} f_{1} \bar{\rho}\left(\frac{\varepsilon}{k}\right) \overline{u_{i} u_{j}} \frac{\partial U_{i}}{\partial x_{j}} \\
& -C_{2} f_{2} \bar{\rho}\left(\frac{\varepsilon^{2}}{k}\right)+\nu \mu_{t}\left(1-f_{\mu}\right)\left(\frac{\partial^{2} U_{i}}{\partial x_{j} \partial x_{j}}\right)^{2} .
\end{aligned}
$$

The empirical constants and model functions in Eqs. (5)-(7) are presented in Table II. 
TABLE II Empirical constants and model functions in the $k-\varepsilon$ turbulence model

\begin{tabular}{cccccccc}
\hline$C_{\mu}$ & $C_{1}$ & $C_{2}$ & $\sigma_{k}$ & $\sigma_{\varepsilon}$ & $f_{1}$ & $f_{2}$ & $f_{\mu}$ \\
\hline 0.09 & 1.44 & 1.9 & 1.0 & 1.3 & $1+0.28 \exp \left(-R_{t} / 25\right)$ & $1-0.3 \exp \left(-R_{t}^{2}\right)$ & $\left\{1-\exp \left(-R_{\tau} / 26.5\right)\right\}^{2}$ \\
\hline
\end{tabular}

TABLE III Empirical constants and model functions in the $\overline{t^{2}}-\varepsilon_{t}$ heat transfer model

\begin{tabular}{cccccccccccc}
\hline$C_{\lambda}$ & $C_{\mathrm{P} 1}$ & $C_{\mathrm{P} 2}$ & $\sigma_{h}$ & $\sigma_{\phi}$ & $C_{\mathrm{D} 1}$ & $C_{\mathrm{D} 2}$ & $f_{\mathrm{P} 1}$ & $f_{\mathrm{P} 2}$ & $f_{\mathrm{D} 1}$ & $f_{\mathrm{D} 2}$ & $f_{\lambda}$ \\
\hline 0.11 & 1.80 & 0.72 & 1.0 & 1.0 & 2.20 & 0.80 & 1.0 & 1.0 & 1.0 & 1.0 & $\left\{1-\exp \left(-(\sqrt{\operatorname{Pr}} / 30.5)(2 / f) \operatorname{St} y^{+}\right)\right\}$ \\
\hline
\end{tabular}

The turbulent heat flux $-c_{p} \bar{\rho} \overline{u_{i} t}$ in Eq. (3) can be given using Boussinesq approximation

$$
-c_{p} \bar{\rho} \overline{u_{i} t}=\lambda_{t} \frac{\partial T}{\partial x_{i}}
$$

Nagano and Kim (1988) expressed the turbulent thermal conductivity $\lambda_{t}$ in terms of the temperature variance, $\overline{t^{2}}$, the dissipation rate of temperature fluctuations, $\varepsilon_{t}, k$ and $\varepsilon$, as

$$
\lambda_{t}=c_{p} \bar{\rho} C_{\lambda} f_{\lambda} k \sqrt{\frac{k \overline{t^{2}}}{\varepsilon \varepsilon_{t}}}
$$

where $C_{\lambda}$ is a model constant and $f_{\lambda}$ is a model function. The two-equation heat transfer model developed by Nagano and Kim (1988) is modified by Torii and Yang (1997a) in order to reproduce the thermal transport characteristics in the turbulent-to-laminar transition region. In the present study, the modified model is used to obtain $\overline{t^{2}}$ and $\varepsilon_{t}$ in Eq. (9). The transport equations for $\overline{t^{2}}$ and $\varepsilon_{t}$ are expressed in tensor form as:

$$
\begin{aligned}
\bar{\rho} U_{j} \frac{\partial \overline{t^{2}}}{\partial x_{j}}= & \frac{\partial}{\partial x_{j}}\left\{\left(\frac{\lambda}{c_{p}}+\frac{\lambda_{t}}{c_{p} \sigma_{h}}\right) \frac{\partial \overline{t^{2}}}{\partial x_{j}}\right\}-2 \bar{\rho} \overline{u_{j} t} \frac{\partial T}{\partial x_{j}} \\
& -2 \bar{\rho} \varepsilon_{t}-2 \frac{\lambda}{c_{p}}\left(\frac{\partial \sqrt{\overline{t^{2}}}}{\partial x_{j}}\right)^{2},
\end{aligned}
$$

and

$$
\begin{aligned}
\bar{\rho} U_{j} \frac{\partial \varepsilon_{t}}{\partial x_{j}}= & \frac{\partial}{\partial x_{j}}\left\{\left(\frac{\lambda}{c_{p}}+\frac{\lambda_{t}}{c_{p} \sigma_{\phi}}\right) \frac{\partial \varepsilon_{t}}{\partial x_{j}}\right\} \\
& -C_{\mathrm{P} 1} f_{\mathrm{P} 1} \bar{\rho}\left(\frac{\varepsilon}{k}\right) \overline{u_{j} t} \frac{\partial T}{\partial x_{j}} \\
& -C_{\mathrm{P} 2} f_{\mathrm{P} 2} \bar{\rho}\left(\frac{\varepsilon_{t}}{k}\right) \overline{u_{i} u_{j}} \frac{\partial U_{i}}{\partial x_{j}} \\
& -C_{\mathrm{D} 1} f_{\mathrm{D} 1} \bar{\rho}\left(\frac{\varepsilon_{t}^{2}}{\overline{t^{2}}}\right)-C_{\mathrm{D} 2} f_{\mathrm{D} 2} \bar{\rho}\left(\frac{\varepsilon \varepsilon_{t}}{k}\right) \\
& +\frac{\alpha \lambda_{t}}{c_{p}}\left(1-f_{\lambda}\right)\left(\frac{\partial^{2} T}{\partial x_{j} \partial x_{j}}\right)^{2^{\prime}},
\end{aligned}
$$

respectively. The empirical constants and model functions in Eqs. (9)-(11) are summarized in Table III. In the present study, a combination of the $k-\varepsilon$ model for velocity field and the $\overline{t^{2}}-\varepsilon_{t}$ model for thermal field is applied to analyze gas flows in a strongly heated pipe parallel to a rotating axis, because the models employed here can predict laminarizing flows in a stationary pipe with high heating (Torii and Yang, 1997a).

A set of governing equations are discretized using the control volume finite-difference procedure developed by Patankar (1980) wherein the control volumes such as the temperature were staggered from those for the velocities. Figure 2 depicts an arrangement of finite-difference variables in the $r-\theta$ cross section. The subscript corresponds to the cell location. The faces of the temperature (i.e. scalar) control volumes coincide 


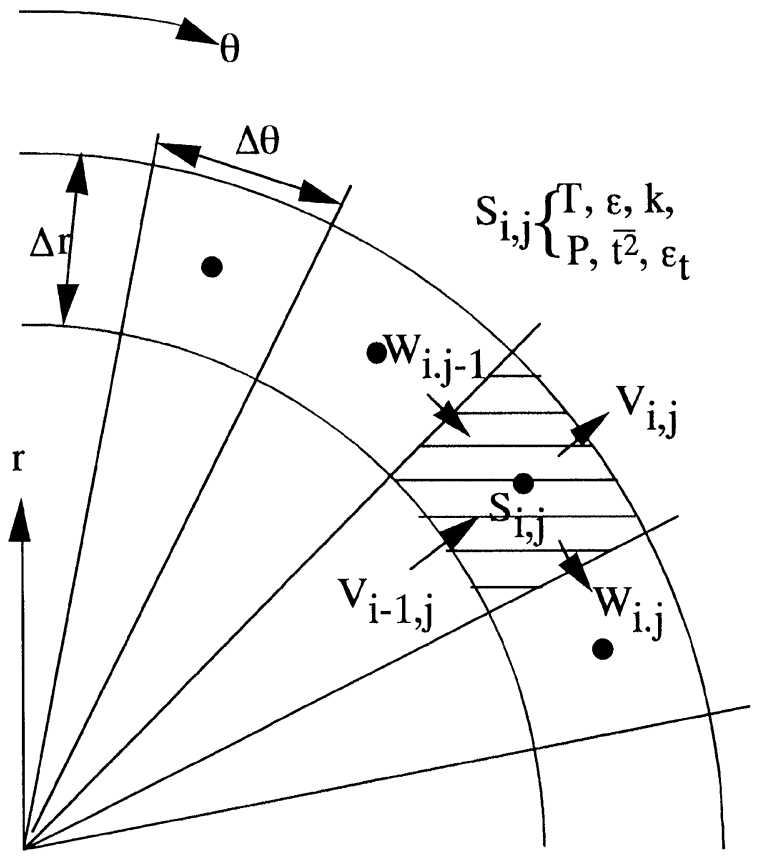

FIGURE 2 Grid arrangement in the $r-\theta$ plane.

with the interfaces of dissimilar materials. Power law variation was used for the interpolation of the dependent variables.

Since all turbulent quantities as well as the timeaveraged velocities vary rapidly in the near-wall region, the size of nonuniform cross-stream grids is increased with a geometric ratio from the wall towards the center line, i.e. the $y$ direction. The maximum control volume size near the center line is always kept less than $3 \%$ of tube radius. Throughout numerical calculations, the number of control volumes is properly selected between $62 \times 62$ and $98 \times 98$ nonuniform grid to obtain a grid-independent solution, resulting in no appreciable difference between the numerical results with different grid spacing. The discritized equations were solved iteratively using the line-by-line TDMA (Tri-Diagonal Matrix Algorithm) and the SIMPLE procedure. The calculations are progressed from the inlet in the downstream direction by means of a marching procedure, because the diffusion terms of momentum and energy equations in the streamwise direction are deleted due to the strong inertia effect. The maximum step-size in the streamwise direction is limited to five times the minimum size in the radial direction of the control volume. At each axial location, the thermal properties for respective control volumes are determined from the axial pressure and temperature by using a numerical code of reference (PROPATH, 1987).

The hydrodynamically, fully-developed, isothermal flow is assumed at the starting point of the heating section. The following boundary conditions are used at the wall:

$$
\begin{aligned}
& r=R \text { (at wall): } \quad U=V=W \\
& =k=\varepsilon=\overline{t^{2}}=\varepsilon_{t}=0, \\
& \frac{\partial T}{\partial r}=\frac{q_{\mathrm{w}}}{\lambda_{\mathrm{w}}} \text { (constant heat flux). }
\end{aligned}
$$

The computations are processed in the following order:

1. Specify the initial values of $U, V, W, k, \varepsilon, T, \overline{t^{2}}$ and $\varepsilon_{t}$ and assign a constant axial pressure gradient. Here, the values of $U, k$ and $\varepsilon$ in the hydrodynamically, fully-developed, isothermal circular tube flow are employed as the initial ones.

2. Solve the equations of $U, k, \varepsilon, T, \bar{t}^{2}$ and $\varepsilon_{t}$.

3. Repeat step 2 until the criterion of convergence is satisfied, which is set at

$$
\max \left|\frac{\phi^{M}-\phi^{M-1}}{\phi_{\max }^{M-1}}\right|<10^{-4}
$$

for all the variables $\phi\left(U, k, \varepsilon, T, \overline{t^{2}}\right.$ and $\left.\varepsilon_{t}\right)$. The superscripts $M$ and $M-1$ in Eq. (12) indicate two successive iterations, while the subscript "max" refers to a maximum value over the entire field of iterations.

4. Calculate new values of $U, k, \varepsilon, T, \overline{t^{2}}$ and $\varepsilon_{t}$ by correcting the axial pressure gradient.

5. Repeat steps 2-4 until the conservation of the streamwise flow rate is satisfied under the criterion

$$
\left|\frac{\iint U_{\text {cp }} \mathrm{d} \theta \mathrm{d} r-\iint U_{\text {in }} \mathrm{d} \theta \mathrm{d} r}{\iint U_{\text {in }} \mathrm{d} \theta \mathrm{d} r}\right| \leq 10^{-5},
$$


and evaluate the convergent values of $U, k$ and $\varepsilon$. Here, $U_{\mathrm{cp}}$ is the axial velocity under the correction process and $U_{\text {in }}$ is that at the inlet of the circular tube.

6. Calculate $V$ and $W$ using the updated values of $U, k, \varepsilon, T, \overline{t^{2}}$ and $\varepsilon_{t}$ until the criterion of convergence, Eq. (12), is satisfied. Here, variables $\phi$ in Eq. (12) correspond to $V$ and $W$.

7. Repeat steps 2-6 until $x$ reaches the desired length, i.e. a location $150 \mathrm{D}$ downstream from the inlet.

In the present study, the nondimensional heat flux parameter, $q_{\mathrm{in}}^{+}$, is employed to indicate the magnitude of heat flux at the tube wall. The ranges of the parameters are nondimensional heat flux parameter $q_{\text {in }}^{+}<0.005$; inlet Reynolds number, i.e. Reynolds number at the onset of heating $\mathrm{Re}_{\text {in }}=8500$; rotation speed of $0-500 \mathrm{rev} / \mathrm{min}$; inlet gas (nitrogen) temperature $T_{\text {in }}=273 \mathrm{~K}$. Numerical computations were performed on a NEC personal computer (32 bit).

In order to verify the $k-\varepsilon$ turbulence and the two-equation heat transfer models employed here and to determine the reliability of the computer code, heat transfer coefficients are calculated. The model is applied to a flow in a circular tube revolving about a horizontal axis parallel to the tube axis, which is heated with a low uniform wall heat flux. Thus, there is no effect of the variation of the gas properties on the velocity and thermal fields. A numerical result is obtained in both the thermal entrance and thermally and hydrodynamically fully-developed regions of a circular tube.

Figure 3, for $\mathrm{Re}=10000$, depicts the streamwise distribution of the Nusselt number in the form of $\mathrm{Nu}$ versus $x / D$ with rotational Reynolds number, $J$, as the parameter. The results are compared with the experimental data of Humphreys et al. (1967). It is observed that the Nusselt number decreases monotonically along the flow due to the thermal entrance effect, and an amplification in local Nusselt number yields due to parallel rotation of the tube. This trend becomes larger with an increase in rotation speed, $J$. The model employed here predicts

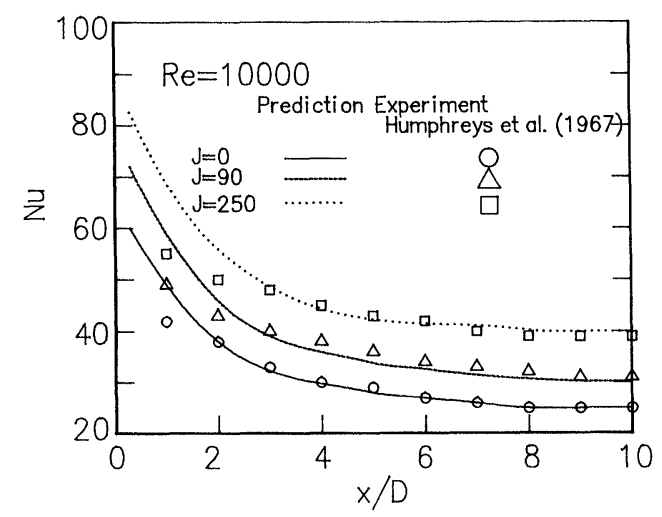

FIGURE 3 A comparison of theoretical and experimental results for the local Nusselt number in the developing tube flows at different rotational Reynolds numbers for the lower heat flux case.

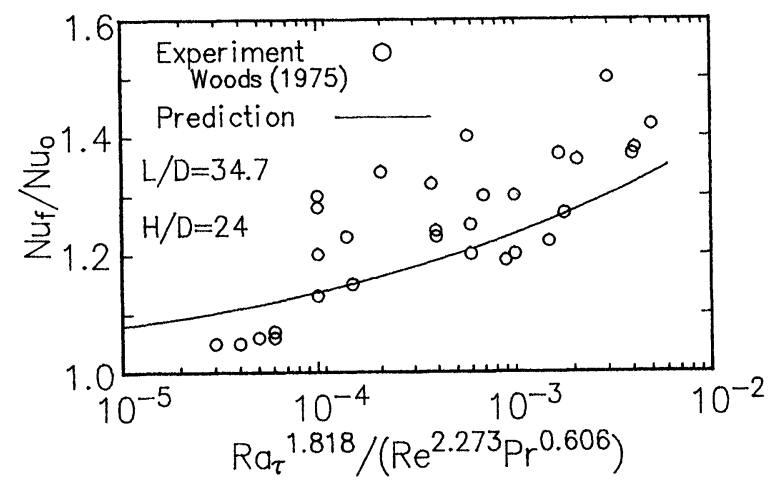

FIGURE 4 A comparison of theoretical and experimental results for the Nusselt number in the fully-developed tube flows at different rotational Rayleigh numbers for the lower heat flux case.

precisely the streamwise variation of the Nusselt number, although the Nusselt number is somewhat overestimated in the vicinity of the inlet.

Figure 4 compares the numerical result with the experimental data (air) of Woods (1975), which are measured at $L / D=34.7$ and $H / D=24$. Here, $\mathrm{Nu}_{0}$ denotes the Nusselt number for a fully-developed turbulent flow in a stationary tube and $\mathrm{Nu}_{\mathrm{f}}$ implies that for a fully developed turbulent flow in a tube rotating around a parallel axis. One observes that the calculated values of Nusselt number are in accord with the experimental data and with $\mathrm{Re}$ fixed, the Nusselt number is amplified with an increase in the rotational Rayleigh number, $\operatorname{Ra}_{\tau}$. In 
other words, heat transfer performance is induced due to the centripetal buoyancy force because the rotational Rayleigh number emerges from the centripetal buoyancy terms of the momentum equations.

To the authors' knowledge, although there is no experimental data pertinent to the turbulence quantity, it is of interest to give the numerical results in the velocity and thermal fields. Figure 5 illustrates the calculated turbulent kinetic energy for a fully-developed turbulent flow in a rotating tube at three different rotational Rayleigh number $\mathrm{Ra}_{\tau}=0,3.8 \times 10^{2}$ and $1.5 \times 10^{3}$. Note that Fig. 5 depicts the radial distribution along $\mathrm{A}-\mathrm{A}^{\prime}$ line in Fig. 1. $\mathrm{Ra}_{\tau}=0$ corresponds to no parallel rotation case. Here, the Reynolds number is fixed at $\mathrm{Re}=10,000$ and numerical results are divided by the square of the friction velocity on the wall in the absence of parallel rotation $\left(u^{*}\right)^{2}$. One observes that as the rotational Rayleigh number, i.e. rotation speed is increased, the turbulent kinetic energy level is enhanced over the entire flow cross section, particularly in the vicinity of the wall. This enhancement yields due to the secondary flow caused by parallel rotation. The corresponding radial distribution of the temperature variance, $\overline{t^{2}}$, in the thermal field is illustrated in Fig. 6. Here, it is normalized by the square of the friction temperature in a fully-developed turbulent flow with a

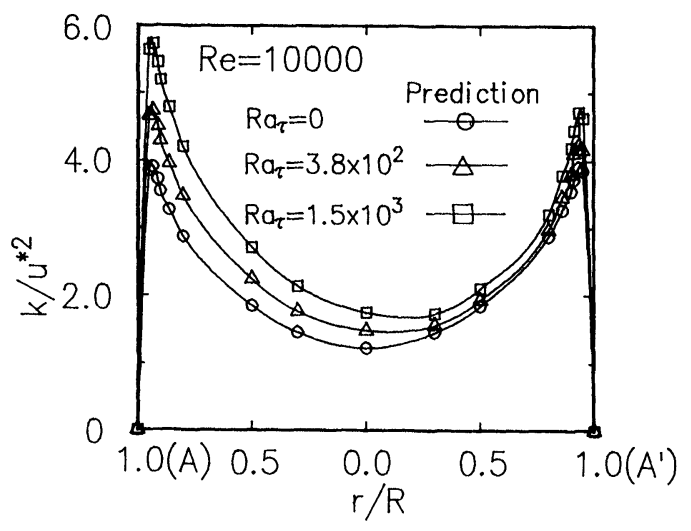

FIGURE 5 Radial distributions of predicted turbulent kinetic energy in the fully-developed tube flows at different rotational Rayleigh numbers for $\mathrm{Re}=10,000$

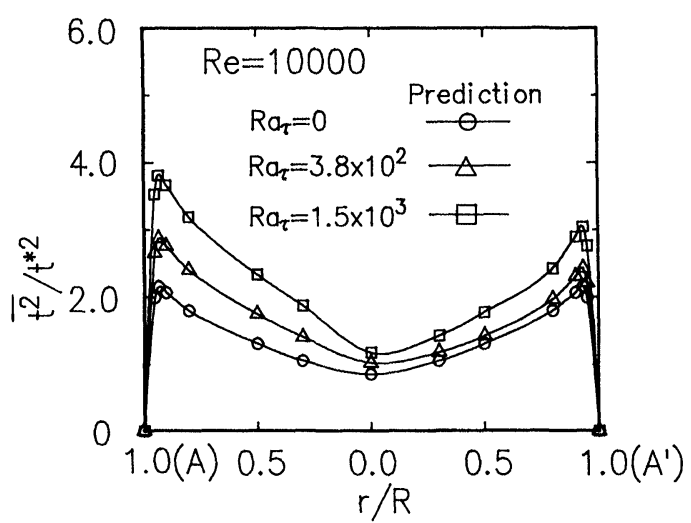

FIGURE 6 Radial distributions of predicted temperature variance in the fully-developed tube flows at different rotational Rayleigh numbers for $\mathrm{Re}=10,000$.

stationary tube, $t^{*}$. The predicted $\overline{t^{2}}$ undergoes a sharp rise in the wall region followed by a gradual decline toward the central region. The temperature variance is intensified with an increase in rotation speed. These behavior is in accord with the variation of heat transfer performance. In other words, a change in heat transfer corresponds to the variations of both the turbulent kinetic energy and the temperature variance. Since the eddy diffusivity concept (Eq. (8)) is employed to determine the turbulent heat flux, $-\overline{u_{i} t}$ in Eq. (3), it is directly related to $k, \varepsilon, \overline{t^{2}}$ and $\varepsilon_{t}$ in Eq. (9). Hence, the amplification in both the turbulent kinetic energy and the temperature variance causes an increase in the Nusselt number, as shown in Fig. 4.

In the above numerical results, the validity of the computer code and the accuracy for $k-\varepsilon$ turbulence and two-equation heat transfer models are borne out.

\section{RESULTS AND DISCUSSION}

Figure 7 illustrates the local heat transfer coefficients in the strongly heated gas flow in the form of Stanton number $\mathrm{St}_{\mathrm{b}}$ versus Reynolds number $\mathrm{Re}_{\mathrm{b}}$, with $q_{\mathrm{in}}^{+}$as the parameter. Numerical results (solid symbols) are compared with the experimental data (hollow symbols) of Bankston (1970), 
which are obtained in the stationary pipe flow. Figures 7(a)-(c) correspond to the rotational Reynolds number, $J$ of 0,95 and 190, respectively. $J=0$ corresponds to no parallel rotation case. The inlet bulk Reynolds number is fixed at 8,500 . Both the Dittus-Boelter's turbulent heat transfer

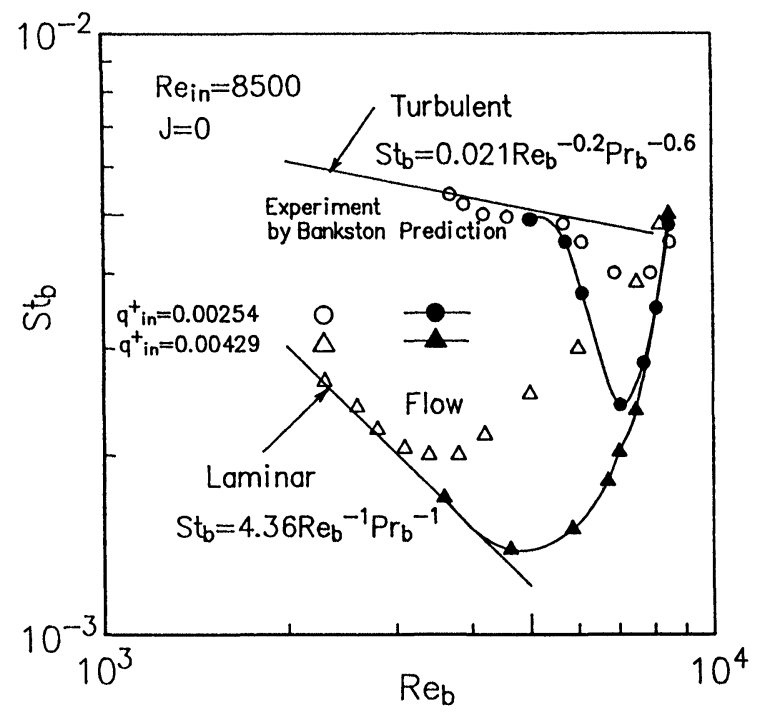

(a)

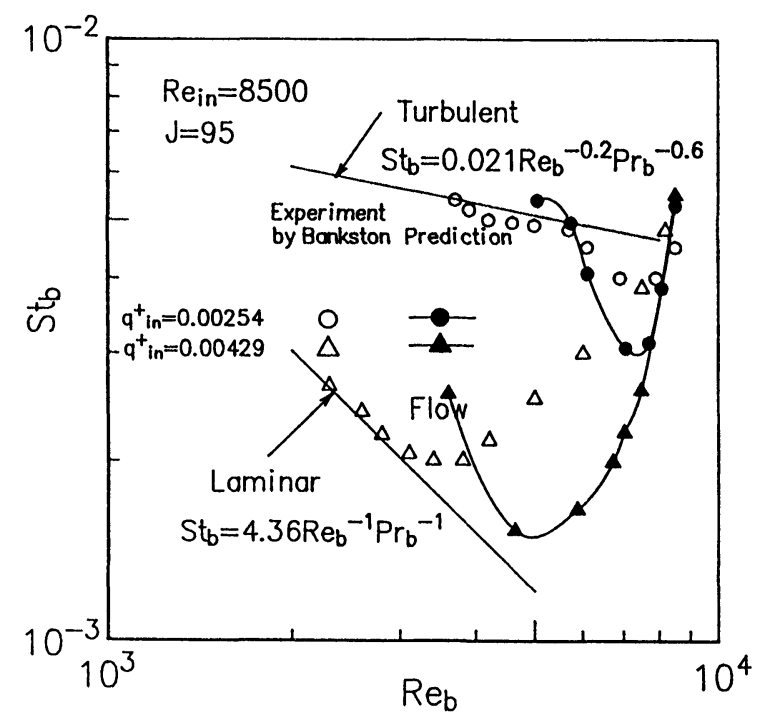

(b)

FIGURE 7(a) and (b)

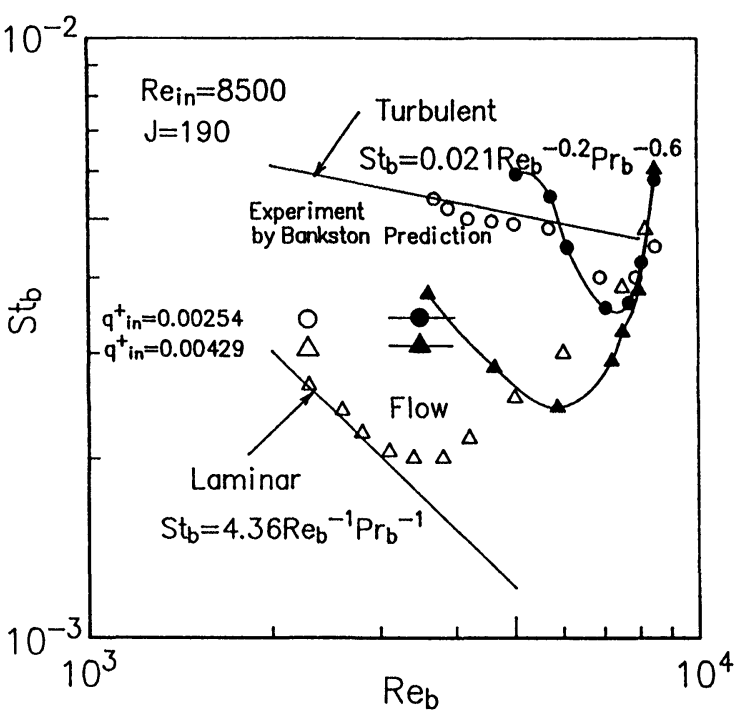

(c)

FIGURE 7(c)

FIGURE 7 Variations of predicted local Stanton number $\mathrm{St}_{\mathrm{b}}$ with Reynolds number $\mathrm{Re}_{\mathrm{b}}$ as a function of nondimensional heat flux $q_{\text {in }}^{+}$for (a) $J=0$, (b) $J=95$ and (c) $J=190$.

correlation and the Stanton number for laminar flow heat transfer in the stationary pipe heated with uniform wall heat flux are superimposed in the figure by solid straight lines. In Fig. 7, a reduction in the bulk Reynolds number implies a change in the streamwise location, because the bulk Reynolds number decreases from the inlet with the axial distance resulting from an increase in the molecular viscosity by heating. It is observed in Fig. 7(a) that at $q_{\text {in }}^{+}=0.00254$, the measured Stanton numbers decrease first in the thermal entrance region, followed by an upturn, approaching the turbulent correlation further downstream. This implies that no laminarization will occur. On the contrary, the experimental Stanton numbers at $q_{\mathrm{in}}^{+}=0.00429$ depart from the turbulent correlation toward the laminar case as the flow goes downstream. Bankston reported that the substantial reduction in $\mathrm{St}_{\mathrm{b}}$ is attributed to the occurrence of laminarization. For $q_{\text {in }}^{+}=0.00254$ and 0.00429 , the turbulence model employed here reproduces the streamwise variation of the experimental data. Figure 7(b), for 
$J=95$, shows that the local Stanton number ceases to decrease after the initial reduction, and begins to recover in the case of $q_{\mathrm{in}}^{+}=0.00429$. This trend is induced with an increase in pipe rotation, as seen in Fig. 7(c) for $J=190$. That is, the local Stanton number finally approaches the turbulent correlation even at higher heating level $\left(q_{\text {in }}^{+}=0.00429\right)$. In other words, as rotation speed, $J$, is increased, no laminarization takes place even at higher heating level $q_{\mathrm{in}}^{+}$, whose level causes the laminarizing flow in the stationary tube. Hence, one may postulate that with high flux heating, a parallel rotation of the pipe induces an amplification in heat transfer performance, that is, the presence of pipe rotation suppresses the promotion of laminarization of gas flow.

An attempt is made to explore the effects of parallel rotation of the tube on the flow and thermal fields, i.e. radial distributions of turbulent kinetic energy, streamwise velocity, and temperature variance along $\mathrm{A}-\mathrm{A}^{\prime}$ line in Fig. 1. Numerical results are obtained at $q_{\text {in }}^{+}=0.00429$, whose heating level causes laminarization in a stationary pipe.

Figure 8 illustrates the effects of rotation speed, $J$, on the radial distributions of the normalized time-averaged streamwise velocity $U / U_{\max }$ at three

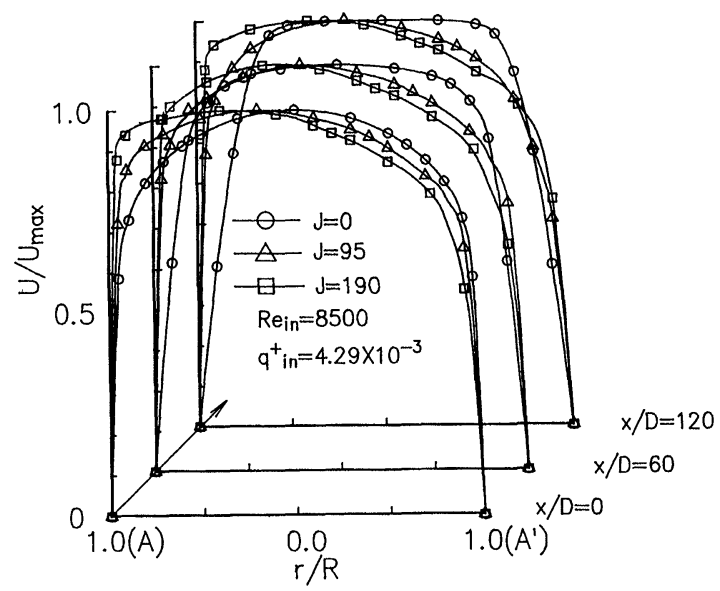

FIGURE 8 Effects of rotational Reynolds number on the distribution of time-averaged streamwise velocity $U / U_{\max }$ in a rotating pipe at three different axial locations, $x / D=0,60$ and 120 , for $\mathrm{Re}_{\text {in }}=8500$ and $q_{\text {in }}^{+}=0.00429$. different axial locations $x / D=0,60$ and 120 . The velocity $U$ is normalized by the maximum value $U_{\max }$ at each axial location. At $J=0$, the velocity gradient at the wall is substantially diminished along the flow by the effect of acceleration due to gas expansion. The acceleration effect suppresses the velocity gradient at the wall even in the case of parallel rotation. This trend becomes minor with an increase in rotation speed, because of the secondary flow caused by parallel rotation. The corresponding streamwise variation of the turbulent kinetic energy is illustrated in Fig. 9. Here, the turbulent kinetic energy $k$ is normalized by a square of the wall friction velocity in the absence of parallel rotation at the onset of heating $u^{*^{2}}$. One observes that the turbulent kinetic energy level at $J=0$ is substantially diminished over the whole tube cross section in the flow direction. The streamwise variation corresponds to an attenuation in the velocity gradient at the wall, as seen in Fig. 8 . The reduction is suppressed by the presence of parallel rotation of the pipe and the turbulent kinetic energy level is maintained in the flow

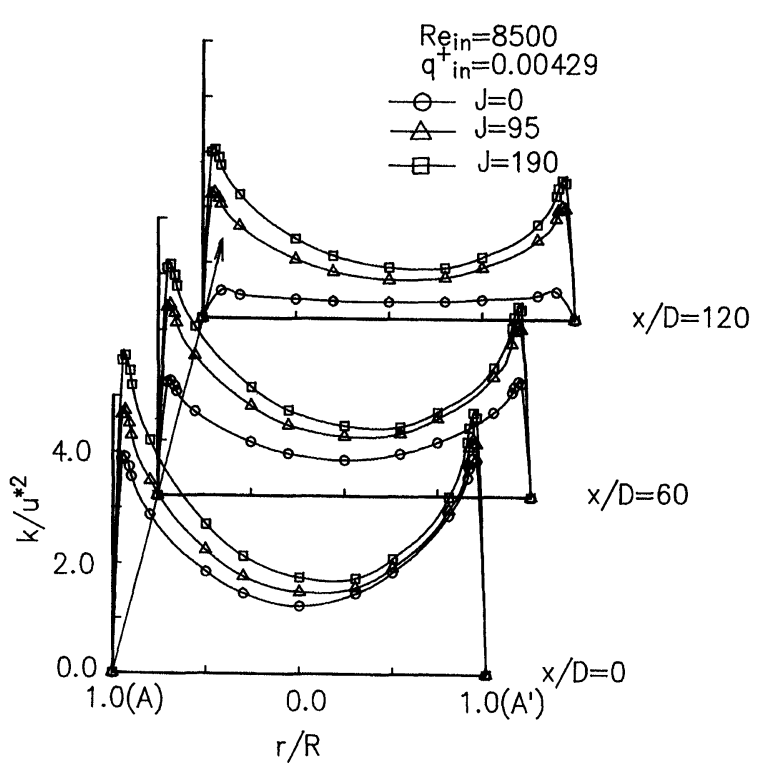

FIGURE 9 Effects of the rotational Reynolds number on the distribution of turbulent kinetic energy $\mathrm{k} / \mathrm{u}^{* 2}$ in a rotating pipe at three different axial locations, $x / D=0,60$ and 120 , for $\mathrm{Re}_{\mathrm{in}}=8500$ and $q_{\mathrm{in}}^{+}=0.00429$. 
direction, in accordance with the variation of the streamwise velocity distribution in Fig. 8.

Figure 10 depicts the radial distribution of the temperature variance, $\overline{t^{2}}$, in the thermal field at the three different axial locations. Here, the temperature variance $\overline{t^{2}}$ is divided by the square of the friction temperature, $t^{*}$, at each axial location. The production of $\overline{t^{2}}$, at $J=0$, is substantially attenuated over the whole pipe cross section in the flow direction. In contrast, $\overline{t^{2}}$ at $J=95$ and 190 is maintained along the flow direction, although the level is slightly diminished in the initial stage. As mentioned previously, the turbulent heat flux, $-\overline{u_{i} t}$ in Eq. (3) is directly related to $k, \varepsilon, \overline{t^{2}}$ and $\varepsilon_{t}$ in Eq. (9), because it is obtained using the eddy diffusivity concept (Eq. (8)). Hence, the presence in the turbulent kinetic energy and the temperature variance suppresses a substantial attenuation in the local Stanton number, as shown in Fig. 7.

In summary, under a very high heat flux heating, reductions of the turbulent kinetic energy, streamwise velocity, and temperature variance during laminarization in a stationary pipe are substantially suppressed due to parallel rotation of the pipe, resulting in the maintenance of heat transfer performance.

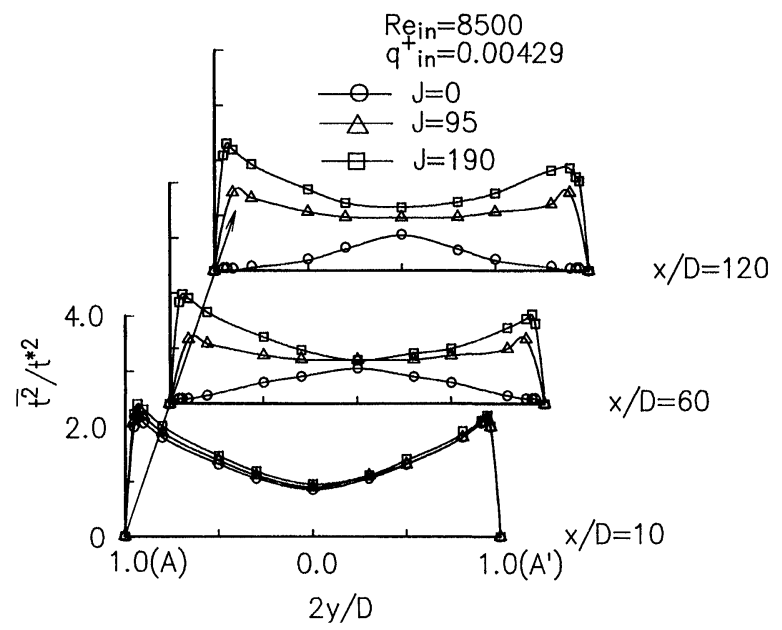

FIGURE 10 Effects of the rotational Reynolds number on the distribution of temperature variance $\overline{t^{2}} / t^{* 2}$ in a rotating pipe at three different axial locations, $x / D=10,60$ and 120 , for $\mathrm{Re}_{\mathrm{in}}=8500$ and $q_{\mathrm{in}}^{+}=0.00429$.

\section{SUMMARY}

A $\overline{t^{2}}-\varepsilon_{t}$ heat transfer model and a $k-\varepsilon$ turbulence model have been employed to numerically investigate fluid flow and heat transfer in a strongly heated gas flow pipe in the presence of its parallel rotation. The study has concluded that:

1. In the higher heat flux case in which laminarization occurs in the stationary pipe flow, a substantial reduction in the local Stanton number is suppressed by parallel rotation of a pipe. Heat transfer performance is maintained with an increase in the rotation speed. In other words, an increase in pipe rotation results in the suppression of laminarization of gas flow.

2. When laminarization is suppressed due to pipe rotation even at higher heat flux case, the velocity gradient in the vicinity of the tube wall is not substantially affected by acceleration due to gas expansion, resulting in a presence in the turbulent kinetic energy over the entire tube cross section. In addition, the temperature variance is also maintained over the whole tube cross section in the flow direction.

\section{NOMENCLATURE}

$$
\begin{aligned}
& c_{p} \\
& C_{\mu}, C_{1}, C_{2} \\
& C_{\lambda}, C_{\mathrm{P} 1}, C_{\mathrm{P} 2}, C_{\mathrm{D} 1}, C_{\mathrm{D} 2} \\
& D \\
& f \\
& f_{\mu}, f_{1}, f_{2} \\
& f_{\lambda}, f_{\mathrm{P} 1}, f_{\mathrm{P} 2}, f_{\mathrm{D} 1}, f_{\mathrm{D} 2} \\
& F \\
& G
\end{aligned}
$$

specific heat at constant pressure, $\mathrm{J} /(\mathrm{kg} \mathrm{K})$ empirical constants of $k-\varepsilon$ model turbulence mode temperature field tube diameter, $\mathrm{m}$ friction coefficient turbulence model functions of $k-\varepsilon$ model turbulence model functions of temperature field third term in Eq. (2) mass flux of gas flow, $\mathrm{kg} /\left(\mathrm{m}^{2} \mathrm{~s}\right)$ constants for 


\begin{tabular}{|c|c|}
\hline$H$ & eccentricity, $\mathrm{m}$ \\
\hline$J$ & rotational Reynolds number, $\Omega D^{2} / 2 \nu$ \\
\hline$k$ & turbulent kinetic energy, $\mathrm{m}^{2} / \mathrm{s}^{2}$ \\
\hline$L$ & heat length, m \\
\hline $\mathrm{Nu}$ & Nusselt number, $q_{\mathrm{w}} D /\left(T_{\mathrm{w}, \text { avg }}-T_{\mathrm{b}}\right) \lambda$ \\
\hline$P$ & time-averaged pressure, $\mathrm{Pa}$ \\
\hline $\operatorname{Pr}$ & Prandtl number \\
\hline $\operatorname{Pr}_{t}$ & turbulent Prandtl number \\
\hline$q$ & heat flux, $\mathrm{W} / \mathrm{m}^{2}$ \\
\hline$q_{\text {in }}^{+}$ & $\begin{array}{l}\text { dimensionless heat flux parameter, } \\
\qquad q_{\mathrm{w}} /\left(G c_{p} T\right)_{\text {in }}\end{array}$ \\
\hline$r$ & radial coordinate, $\mathrm{m}$ \\
\hline$R$ & tube radius, $D / 2, \mathrm{~m}$ \\
\hline $\mathrm{Ra}_{\tau}$ & $\begin{array}{l}\text { rotational Rayleigh number, } \\
\Omega^{2} H \beta \tau R^{4} / \alpha \nu\end{array}$ \\
\hline $\operatorname{Re}$ & Reynolds number, $u_{\mathrm{m}} D / \nu$ \\
\hline$R_{\mathrm{t}}$ & turbulent Reynolds number, $k^{2} /(\varepsilon \nu)$ \\
\hline $\mathrm{St}$ & $\begin{array}{l}\text { Stanton number, } \\
\qquad q_{\mathrm{w}} /\left(\rho c_{p} u_{\mathrm{m}}\left(T_{\mathrm{w}, \mathrm{avg}}-T_{\mathrm{b}}\right)\right)\end{array}$ \\
\hline$T$ & time-averaged temperature, $\mathrm{K}$ \\
\hline$t$ & $\begin{array}{l}\text { fluctuating temperature } \\
\text { component, } \mathrm{K}\end{array}$ \\
\hline$\frac{t^{*}}{-2}$ & friction temperature, $q_{\mathrm{w}} /\left(\rho c_{p} u^{*}\right), \mathrm{K}$ \\
\hline$\overline{t^{2}}$ & temperature variance, $K^{2}$ \\
\hline$U, V, W$ & $\begin{array}{l}\text { time-averaged velocity components } \\
\text { in axial, radial and tangential } \\
\text { directions, respectively, } \mathrm{m} / \mathrm{s}\end{array}$ \\
\hline$U_{i}$ & time-averaged velocity, $\mathrm{m} / \mathrm{s}$ \\
\hline$u_{i} u_{j}$ & Reynolds stress, $\mathrm{m}^{2} / \mathrm{s}^{2}$ \\
\hline$u_{i} t$ & Turbulent heat flux, $\mathrm{mK} / \mathrm{s}$ \\
\hline$u_{\mathrm{m}}$ & $\begin{array}{l}\text { mean velocity over tube } \\
\text { cross section, } \mathrm{m} / \mathrm{s}\end{array}$ \\
\hline$u^{*}$ & friction velocity, $\mathrm{m} / \mathrm{s}$ \\
\hline$x$ & axial coordinate, $\mathrm{m}$ \\
\hline$x_{i}$ & $i$ coordinate, $\mathrm{m}$ \\
\hline$y$ & distance from wall, $\mathrm{m}$ \\
\hline$y^{+}$ & dimensionless distance, $u^{*} y / \nu$ \\
\hline
\end{tabular}

$\rho$
$\varepsilon$
$\varepsilon_{t}$
$\lambda, \lambda_{t}$

$\mu, \mu_{t}$
$\nu$
$\sigma_{k}, \sigma_{\varepsilon}, \sigma_{h}, \sigma_{\phi}$
$\tau$
$\theta$
$\Omega$

\section{Subscripts}

$\begin{array}{ll}\text { avg } & \text { average } \\ \mathrm{b} & \text { bulk } \\ \text { in } & \text { inlet } \\ \max & \text { maximum } \\ \text { w } & \text { wall }\end{array}$

\section{Superscripts}

time-averaged value fluctuation value

\section{References}

Bankston, C.A. (1970) The transition from turbulent to laminar gas flow in a heated pipe, Trans. ASME Ser. C, 92, 569-579. Coon, C.W. and Perkins, H.C. (1970) Transition from the turbulent to the laminar regime for internal convective flow with large property variations, Trans. ASME Ser. C, 92, 506-512.

Humphreys, J.F., Morris, W.D. and Barrow, H. (1967) Convection heat transfer in the entry region of a tube which revolves about an axis parallel to itself, Int. J. Heat Mass Transfer, 10, 333-347.

Kataoka, K., Bitou, Y. and Hashioka, K. (1984) Mass transfer in the annulus between two coaxial rotating cylinders, In Heat and Mass Transfer in Rotating Machinery, Eds. D.E. Metzeger and N.H. Afgan, pp. 143-153, Hemisphere, Washington, DC.

Kawamura, H. (1979) Prediction of strongly heated turbulent flow of gas in a circular tube using a two-equation model of turbulence, Trans. Jpn. Soc. Mech. Eng. (in Japanese), 45(395) B, 1038-1046. 
McEligot, D.M., Coon, C.M. and Perkins, H.C. (1970) Relaminarization in tubes, J. Heat and Mass Transfer, 13, 431-433.

Mori, Y. and Nakayama, W. (1984) Secondary flows and enhanced heat transfer in rotating pipes and ducts, Heat and Mass Transfer in Rotating Machinery, Eds. D. E. Metzeger and N.H. Afgan, pp. 3-24, Hemisphere, Washington, DC.

Mori, Y. and Nakayama, W. (1968) Convective heat transfer in rotating radial circular pipe (1st Report), Int. J. of Heat and Mass Transfer, 11, 1027-1040.

Mori, Y., Fukuda, T. and Nakayama, W. (1971) Convective heat transfer in rotating radial circular pipe (2nd Report), Int. $J$. of Heat and Mass Transfer, 14, 1807-1824.

Mori, Y. and Watanabe, K. (1979) Reduction in heated transfer performance due to high heat flux, Trans. Jpn. Soc. Mech. Eng. (in Japanese), 45(397) B, 1343-1353.

Morris, W.D. (1981) Heat Transfer and Fluid Flow in Rotating Coolant Channels, Research Studies Press, Chichester, UK.

Nagano, Y. and Hishida, M. (1987) Improved form of the $k-\varepsilon$ model for wall turbulent shear flows, Trans. of ASME, Ser. D, 109, 156-160.

Nagano, Y. and Kim, C. (1988) A Two-equation model for heat transport in wall turbulent shear flows, J. Heat Transfer, 110 583-589.

Ogawa, M., Kawamura, H. Takizuka, T. and Akino, N. (1982) Experimental on laminarization of strongly heated gas flow in vertical circular tube, J. Atomic Energy Soc. Jpn. (in Japanese), 24(1), 60-67.

Patankar, S.V. (1980) Numerical Heat Transfer and Fluid Flow, Hemisphere, Washington, DC.
Perkins, K.R., Schade, K.W. and McEligot, D.M. (1973) Heated laminarizing gas flow in a square duct, J. Heat and Mass Transfer, 16, 897-916.

Propath Group (1987) Propath: a Program Package for Thermophysical Property, version 4.1 (in Japanese).

Rodi, W. (1982) Examples of turbulence models for incompressible flows, AIAA, J., 20, 872-879.

Schlichting, H. (1985) Boundary Layer Theory, 2nd ed., McGraw-Hill, New York.

Torii, S., Shimizu, A., Hasegawa, S. and Higasa, M. (1990) Laminarization of strongly heated gas flows in a circular tube (Numerical analysis by means of a modified $k-\varepsilon$ model), JSME Int. J., Ser. II, 33(33), 538-547.

Torii, S., Shimizu, A. and Hasegawa, S. (1993) Numerical analysis of laminarizing tube flows by means of a Reynolds stress turbulence model, Heat Transfer - Japanese Research, 22(2), 154-170.

Torii, S. and Yang, W.-J. (1997a) Laminarization of turbulent gas flows inside a strongly heated tube, Int. J. Heat Mass Transfer, 40(13), 3105-3117.

Torii, S. and Yang, W.-J. (1997b) Effects of rotation on laminarization of gas flow in a strongly heated tube, HTDVol. 353, Proceedings of the ASME Heat Transfer Division, 3 , 225-232.

Woods, J.L. (1975) Heat transfer and flow resistance in a rotating duct system, D.Phil. Thesis, University of Sussex, Falmer, England.

Yang, W.J. and Zhang, N. (1992) Steady thermal and hydrodynamic layer flows with rotation, Transport Phenomena in Heat and Mass Transfer, Ed. J.A. Reizes, Elsevier Science Publishers, pp. 337-351. 


\section{ait \\ ENERGY MATERIALS}

M A N E Y publishing

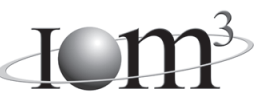

\section{Materials Science \& Engineering for Energy Systems}

Maney Publishing on behalf of the Institute of Materials, Minerals and Mining

The Institute of Materials, Minerals \& Mining

Economic and environmental factors are creating ever greater pressures for the efficient generation, transmission and use of energy. Materials developments are crucial to progress in all these areas: to innovation in design; to extending lifetime and maintenance intervals; and to successful operation in more demanding environments. Drawing together the broad community with interests in these areas, Energy Materials addresses materials needs in future energy generation, transmission, utilisation, conservation and storage. The journal covers thermal generation and gas turbines; renewable power (wind, wave, tidal, hydro, solar and geothermal); fuel cells (low and high temperature); materials issues relevant to biomass and biotechnology; nuclear power generation (fission and fusion); hydrogen generation and storage in the context of the 'hydrogen economy'; and the transmission and storage of the energy produced.

As well as publishing high-quality peer-reviewed research, Energy Materials promotes discussion of issues common to all sectors, through commissioned reviews and commentaries. The journal includes coverage of energy economics and policy, and broader social issues, since the political and legislative context influence research and investment decisions.

\section{CALL FOR PAPERS}

Contributions to the journal should be submitted online at http://ema.edmgr.com

To view the Notes for Contributors please visit: www.maney.co.uk/journals/notes/ema

Upon publication in 2006, this journal will be available via the Ingenta Connect journals service. To view free sample content online visit: www.ingentaconnect.com/content/maney

For further information please contact:

Maney Publishing UK

Tel: +44 (0)113 2497481 Fax: +44 (0)1132486983 Email: subscriptions@maney.co.uk

or

Maney Publishing North America

Tel (toll free): 8662975154 Fax: 6173546875 Email: maney@maneyusa.com

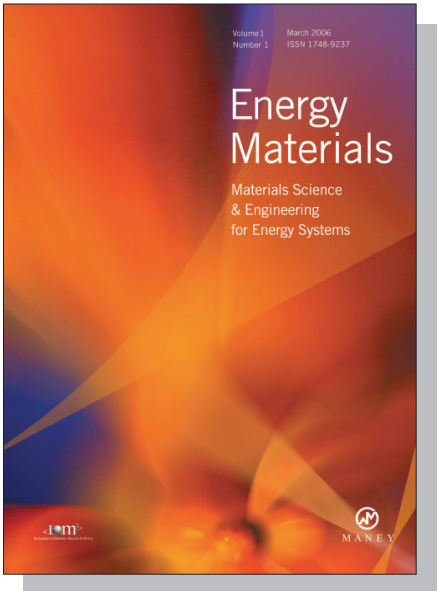

EDITORS

Dr Fujio Abe

NIMS, Japan

Dr John Hald, IPL-MPT, Technical University of Denmark, Denmark

Dr R Viswanathan, EPRI, USA

\section{SUBSCRIPTION INFORMATION}

Volume 1 (2006), 4 issues per year

Print ISSN: 1748-9237 Online ISSN: 1748-9245

Individual rate: $£ 76.00 / U S \$ 141.00$

Institutional rate: $£ 235.00 /$ US $\$ 435.00$

Online-only institutional rate: $£ 199.00 / U S \$ 367.00$

For special $\mathrm{IOM}^{3}$ member rates please email

subscriptions@maney.co.uk

\section{For further information or to subscribe online please visit www.maney.co.uk}



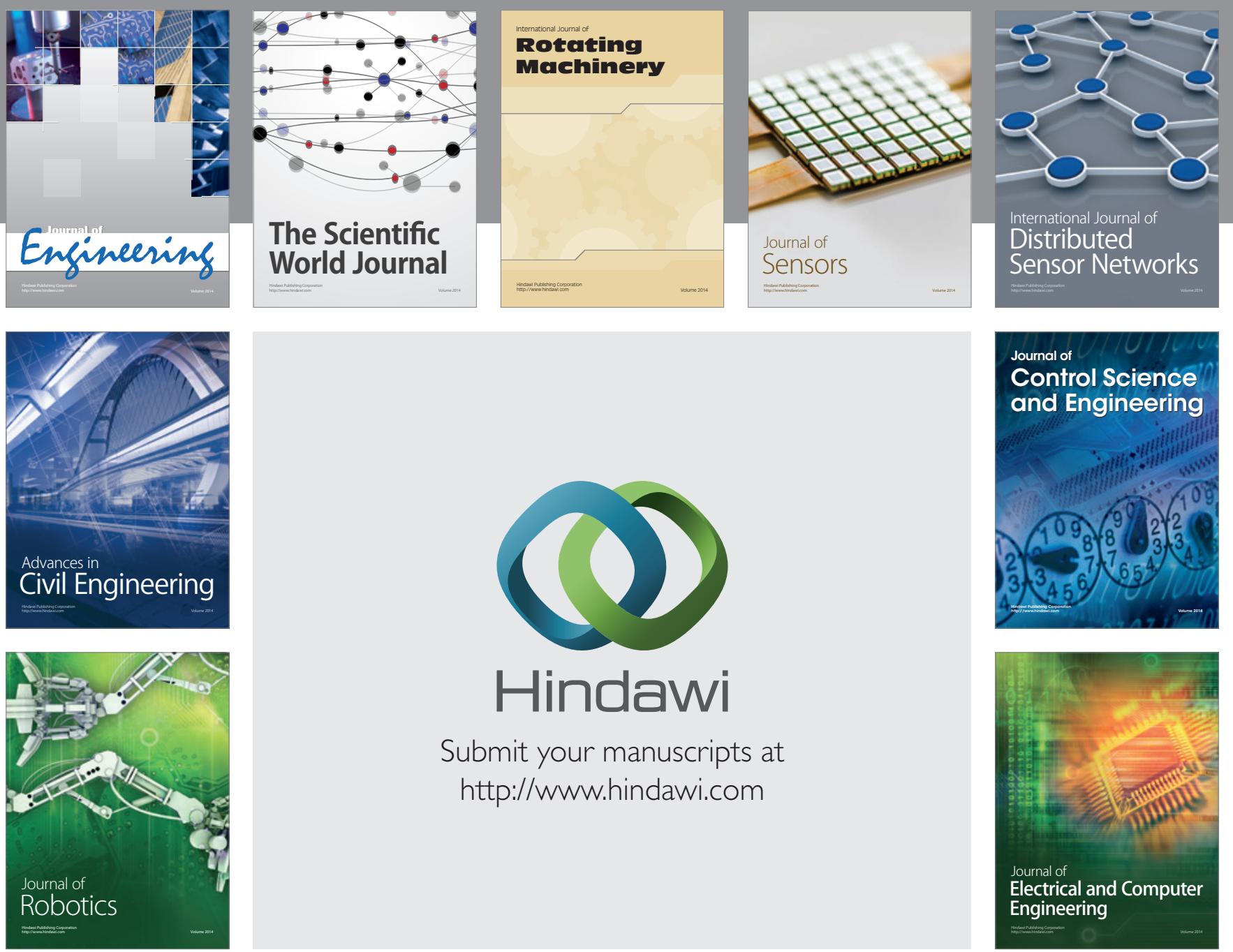

Submit your manuscripts at

http://www.hindawi.com
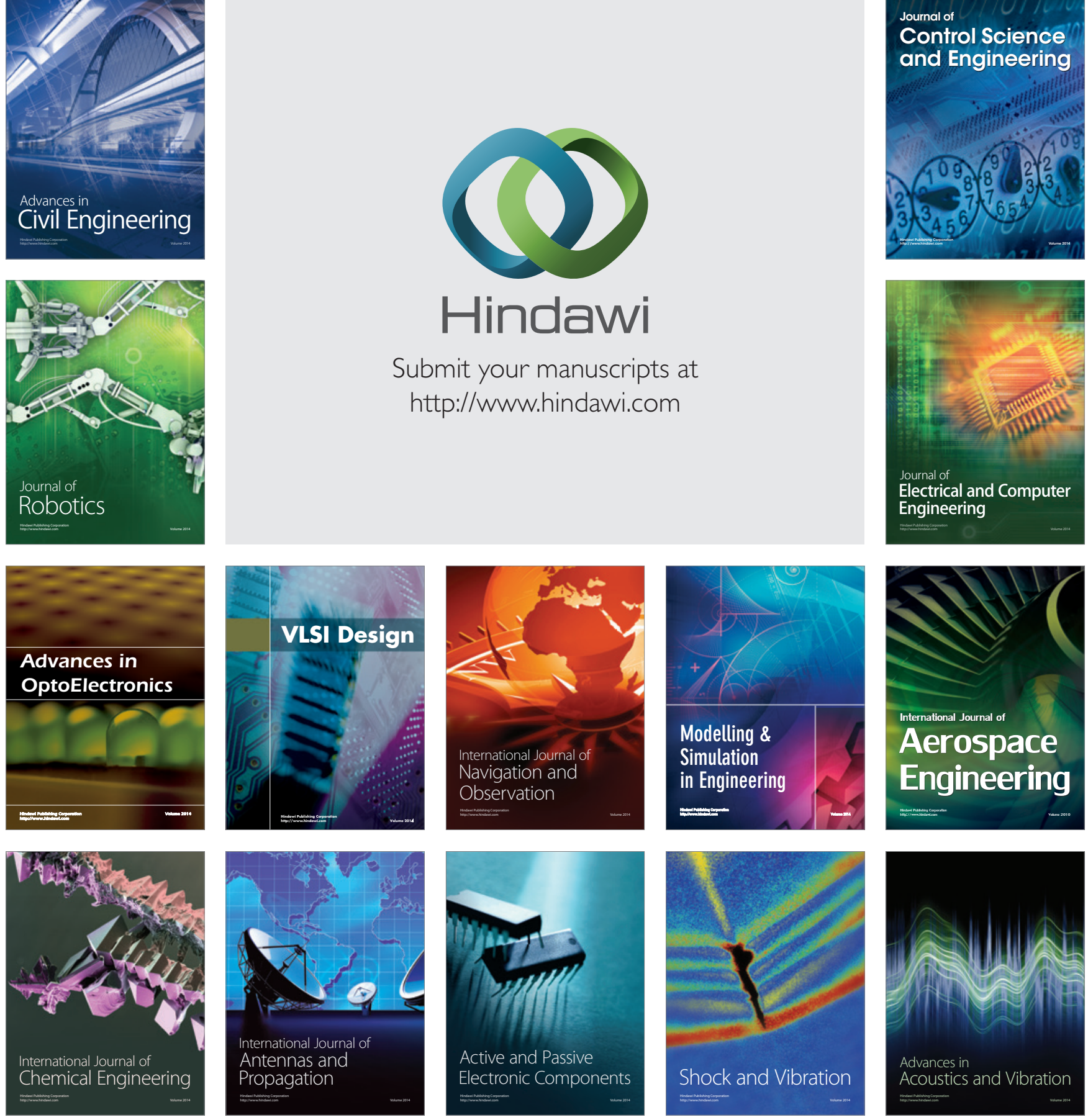